\title{
ON GENERALIZED RESOLVENTS OF SYMMETRIC LINEAR RELATIONS IN A PONTRJAGIN SPACE
}

\author{
PEKKA SORJONEN
}

\section{Introduction}

In [4] we gave a characterization of the generalized resolvents of a symmetric operator with arbitrary defect numbers in a Pontrjagin space $\Pi_{\varkappa}$. The purpose of this note is to extend this and related results to symmetric linear relations in $\Pi_{x}$. As was pointed out in [5], the need for this kind of extension arises e.g. in connection with differential relations with an indefinite weight function.

Because this paper is a continuation of [5], we shall freely use the notions and results from [5].

\section{Generalized resolvents}

Throughout this paper $\mathfrak{H}$ denotes a Pontrjagin space $\Pi_{\varkappa}$ with an (indefinite) inner product $[\cdot \mid \cdot]$ which has $\varkappa$ negative squares, and $\mathfrak{H}^{2}$ is the product space $\mathfrak{H} \oplus \mathfrak{H}$. Furthermore, $T$ always stands for a closed symmetric linear relation in $\mathfrak{H}$; i.e., $T$ is a closed subspace of $\mathfrak{S}^{2}$ with $T \subset T^{+}$, where

$$
T^{+}:=\left\{(h, k) \in \mathfrak{S}^{2} \mid[g \mid h]=[f \mid k] \text { for all }(f, g) \in T\right\} .
$$

A self-adjoint extension $S$ of $T$, i.e. $S^{+}=S \supset T$, is said to be regular if $S \subset \mathfrak{\Re}^{2}$, where $\mathfrak{K} \supset \mathfrak{H}$ is a Pontrjagin space with $\varkappa$ negative squares. Let $S$ be such an extension. The function $R: \varrho(S) \rightarrow \mathscr{B}(\mathfrak{H})$,

$$
R(z):=\left.P(S-z I)^{-1}\right|_{\mathfrak{S}} \quad(z \in \varrho(S)),
$$

is called a generalized resolvent of $T$; here $P$ denotes the orthogonal projector of $\mathfrak{K}$ onto $\mathfrak{H}$. If in addition $S$ extends a maximal symmetric relation $T^{\prime}$ in $\mathfrak{H}$ with the upper defect number $n_{+}\left(T^{\prime}\right)=0$ (resp. lower defect number $n_{-}\left(T^{\prime}\right)=0$ ), then $R$ is upper canonical (resp. lower canonical).

We suppose that the domain $\mathcal{D}(T)$ of $T$ includes the negative component $\mathfrak{H}_{-}$of a fundamental decomposition of $\mathfrak{H}$. Then $T$ has regular self-adjoint extensions ([5], Corollary 4.7), and there exists a constant $c>0$ such that the spaces $\mathfrak{N}_{z}:=\mathfrak{R}(T-\bar{z} I)^{\perp}$ with $|\operatorname{Im} z|>c$ are Hilbert spaces with respect to the indefinite inner product $[\cdot \mid \cdot]$; see [5], Theorem 4.10 . 
Take a fixed complex number $w$ with $\operatorname{Im} w>c$ and denote by $V$ the Cayley transform $C_{w}(T)$ of $T$ :

$$
V:=\{(g-w f, g-\bar{w} f) \mid(f, g) \in T\} .
$$

Then $\mathfrak{N}_{\bar{w}}=\mathfrak{D}(V)^{\perp}$ and $\mathfrak{N}_{w}=\mathfrak{R}(V)^{\perp}$; see [5]. Let $\Gamma_{0}$ (resp. $\Gamma_{\infty}$ ) be the orthogonal projector of $\mathfrak{S}$ onto $\mathfrak{N}_{\bar{w}}$ (resp. $\mathfrak{N}_{w}$ ). The characteristic function of $V$ is defined by the equation

$$
X(\lambda):=\left.\lambda^{-1} \Gamma_{0}\left(I-\lambda V^{\prime}\right)^{-1}\right|_{\mathfrak{R}_{w}} \quad(|\lambda|<1) ;
$$

here $V^{\prime}$ is the zero extension of $V$ :

$$
V^{\prime} f:= \begin{cases}V f & \text { for } f \in \mathcal{D}(V), \\ 0 & \text { for } f \in \mathfrak{N}_{\overline{\mathbf{w}}} .\end{cases}
$$

Then the characteristic function of $T$ is $Y(z):=X\left(\lambda(z)^{-1}\right)$, where $\lambda(z):=(z-\bar{w}) /(z-w)$ for $z$ in the complex plane $C$. Note that $Y$ is a meromorphic function in the open upper half-plane $\boldsymbol{C}_{+}$of $\boldsymbol{C}$ with values in $\mathscr{B}\left(\mathfrak{N}_{w}, \mathfrak{N}_{\bar{w}}\right)$; see [2] or [4].

Let $S$ be a fixed regular self-adjoint extension of $T$ and $R$ the corresponding generalized resolvent. For $z \in \varrho(S)$ we define (see [2])

$$
\begin{gathered}
\Gamma_{+}(z):=\Gamma_{\infty}+(z-w) R(z) \Gamma_{\infty}, \\
\Gamma_{-}(z):=\Gamma_{0}+(z-\bar{w}) R(z) \Gamma_{0}, \\
F(z):=\left.(z-w)^{-1} \Gamma_{\infty}\left\{(z-\bar{w}) I-(w-\bar{w})[I+(z-w) R(z)]^{-1}\right\}\right|_{\mathfrak{P}_{\bar{w}}} .
\end{gathered}
$$

Then $F$ belongs to the class $\mathscr{K}_{+}\left(\mathfrak{N}_{\bar{w}}, \mathfrak{N}_{w}\right)$ of the functions which are holomorphic in $C_{+}$with contractive operator values in $\mathscr{B}\left(\mathfrak{N}_{\bar{w}}, \mathfrak{N}_{w}\right)$. In the following we shall use the phrase "for almost all $z \in \boldsymbol{C}_{+}$" or shortly "for a.a. $z \in \boldsymbol{C}_{+}$" to mean "for all $z \in \boldsymbol{C}_{+}$with the possible exception of a countable set which does not have any cluster points in $\boldsymbol{C}_{+}$".

Theor em 1.1. Let $T$ be a closed symmetric linear relation in a Pontrjagin space $\mathfrak{H}$ with $\mathfrak{D}(T) \supset \mathfrak{H}_{-}$and $\mathfrak{n}_{ \pm}(T)>0$ and let $w$ be a complex number with $\operatorname{Im} w>c$. If $R$ is a given regular generalized resolvent of $T$, then the formula

$$
\tilde{R}(z)=R(z)+(w-\bar{w})^{-1} \Gamma_{+}(z) B(z) \Gamma_{-}(\bar{z})^{+},
$$

where

$$
B(z):=(I-F(z) Y(z))(I-\tilde{F}(z) Y(z))^{-1}(\tilde{F}(z)-F(z))
$$

for almost all $z \in C_{+}$, defines a bijective correspondence between all regular generalized resolvents $\tilde{R}$ of $T$ and all functions $\tilde{F} \in \mathscr{K}_{+}\left(\mathfrak{N}_{\bar{w}}, \mathfrak{N}_{w}\right)$.

Furthermore, $\tilde{R}$ is upper (resp. lower) canonical if and only if $\tilde{F}$ is independent of $z$ and $\widetilde{F}(z)$ (resp. $\left.\widetilde{F}(z)^{+}\right)$is isometric.

This result extends [2], Satz 4.2. The proof follows in the same way as in the operator case via the Cayley transformation; see [2] and [4]. 
In the case of equal defect numbers the characterization of the generalized resolvents of a symmetric linear operator or relation uses the so-called $Q$-function instead of the characteristic function $Y$ and dissipative operators or relations instead of contractive operators $\tilde{F}(z)$; see [1] and [3]. We show that this kind of characterization can be given by use of Theorem 1.1.

For this, let $T$ be as in Theorem 1.1 and suppose that the defect numbers of $T$ are both equal to $\mathfrak{n}$. In this case $T$ has a self-adjoint extension in the original space $\mathfrak{H}$. Indeed, the Cayley transform $V=C_{w}(T)$ of $T$ is an isometric operator with $\operatorname{dim} \mathfrak{D}(V)^{\perp}=\operatorname{dim} \mathfrak{R}(V)^{\perp}=\mathfrak{n}$ and $\mathfrak{D}(V)^{\perp}$ as well as $\mathfrak{R}(V)^{\perp}$ are Hilbert spaces, so that $V$ has a unitary extension in $\mathfrak{H}$. The inverse Cayley transform of this unitary operator is then a self-adjoint extension of $T$ in $\mathfrak{H}$.

Choose a self-adjoint extension $S$ of $T$ in $\mathfrak{H}$ and let $R$ be the corresponding generalized resolvent. Then, by Theorem 1.1, the function $F \in \mathscr{K}_{+}\left(\mathfrak{N}_{\bar{w}}, \mathfrak{N}_{w}\right)$ is independent of $z$ and $F F^{+}=F^{+} F$ with $F:=F(z)$.

To form a $Q$-function of $T$ we first choose a Hilbert space $\mathfrak{b}$ with $\operatorname{dim} \mathfrak{\mathfrak { b }}=\mathfrak{n}$ and a bijective operator $\Gamma \in \mathscr{B}\left(\mathfrak{G}, \mathfrak{N}_{w}\right)$. Define

$$
Q^{\prime}(z):= \begin{cases}\left(C_{-i y}\right)^{-1}(F Y(z)) & \text { for } z \in C_{+}, \\ Q^{\prime}(\bar{z})^{+} & \text {for } z \in C_{-}\end{cases}
$$

here $y:=\operatorname{Im} w$. It is not too difficult to show that the functions $Q(z):=\Gamma^{+} Q^{\prime}(z) \Gamma \in \mathscr{B}(\mathfrak{G})$ and $\Gamma(z):=\Gamma_{+}(z) \Gamma \in \mathscr{B}\left(\mathfrak{F}, \mathfrak{N}_{z}\right)$ satisfy the equation

$$
(z-\bar{\zeta})^{-1}\left(Q(z)-Q(\zeta)^{+}\right)=\Gamma(\zeta)^{+} \Gamma(z) \quad(z, \zeta \in \varrho(S)),
$$

i.e., $Q$ is a $Q$-function of $T$ in the sense of [1].

Let $\tilde{R}$ be an arbitrary regular generalized resolvent of $T$ and $\tilde{F} \in \mathscr{K}_{+}\left(\mathfrak{N}_{\bar{w}}, \mathfrak{N}_{w}\right)$ the function assigned to it by Theorem 1.1. With the function $B$ given in (1.2) we get

$$
\begin{aligned}
& (w-\bar{w})^{-1} B(z) F^{+} \\
& =(2 i y)^{-1}\left\{(I-F Y(z))^{-1}-\tilde{F}(z) Y(z)(I-F Y(z))^{-1}\right\}^{-1}\left(\tilde{F}(z) F^{+}-I\right) \\
& =-\left\{Q^{\prime}(z)+i y I-\tilde{F}(z) F^{+}\left(Q^{\prime}(z)-i y I\right)\right\}^{-1}\left(I-\tilde{F}(z) F^{+}\right) .
\end{aligned}
$$

Define $D^{\prime}(z):=\left(C_{-i y}\right)^{-1}\left(\tilde{F}(z) F^{+}\right)$; then a little calculation gives

$$
Q^{\prime}(z)+D^{\prime}(z)=\left(I-\widetilde{F}(z) F^{+}\right)\left\{Q^{\prime}(z)+i y I-\widetilde{F}(z) F^{+}\left(Q^{\prime}(z)-i y I\right)\right\} .
$$

Furthermore, one can verify that $\Gamma_{-}(\bar{z})^{+}=F^{+}\left(\Gamma^{+}\right)^{-1} \Gamma(\bar{z})^{+}$. Put this and (1.3)(1.4) together to get

$$
\begin{aligned}
(w-\bar{w})^{-1} \Gamma_{+}(z) B(z) \Gamma_{-}(\bar{z})^{+} & =(2 i y)^{-1} \Gamma(z) \Gamma^{-1} B(z) F^{+}\left(\Gamma^{+}\right)^{-1} \Gamma(\bar{z})^{+} \\
& =-\Gamma(z)(Q(z)+D(z))^{-1} \Gamma(\bar{z})^{+},
\end{aligned}
$$

where $D(z):=\Gamma^{+} D^{\prime}(z) \Gamma$.

Denote by $\mathscr{D}_{+}(\mathfrak{5})$ the set of all functions $z \mapsto D(z)$ such that $D(z), z \in C_{+}$, is a maximal dissipative linear relation in $\mathfrak{G}$ and the mapping $z \mapsto C_{-i y}(D(z))$ is 
holomorphic in $C_{+}$. By using the results of [5] one can show that $D \in \mathscr{D}_{+}(\mathfrak{5})$. As the calculations above are invertible we can write the following result, which extends [1], Theorem 5.1 and [3], Theorem 3.2.

Corollary 1.2. Let $T$ be a closed symmetric linear relation in a Pontrjagin space $\mathfrak{H}$ with $\mathfrak{D}(T) \supset \mathfrak{H}_{-}$and $\mathfrak{n}_{+}(T)=\mathfrak{n}_{-}(T)>0$. Let $R$ be a generalized resolvent of $T$ in the original space. The formula

$$
\tilde{R}(z)=R(z)-\Gamma(z)(Q(z)+D(z))^{-1} \Gamma(\bar{z})^{+}
$$

gives a bijective correspondence between the set of all regular generalized resolvents $\tilde{R}$ of $T$ and the set $\mathscr{D}_{+}(\mathfrak{b})$.

\section{Resolvent matrices}

In this section we suppose that $T$ is a closed symmetric linear relation in a Pontrjagin space $\mathfrak{H}$ with $\mathfrak{D}(T) \supset \mathfrak{H}_{-}$and $\mathfrak{n}_{+}(T) \geqq \mathfrak{n}_{-}(T)>0$. Furthermore, let $w$ be a fixed complex number with $\operatorname{Im} w>c$.

Let us take two closed subspaces $\mathfrak{L}_{ \pm}$of $\mathfrak{H}$ with $\operatorname{dim} \mathfrak{L}_{ \pm}=\mathfrak{n}_{ \pm}(T)$. If $P_{ \pm}$ are (not necessarily orthogonal) projectors of $\mathfrak{H}$ onto $\mathfrak{L}_{ \pm}$, then the adjoints $P_{ \pm}^{+}$ are also projectors and $\mathfrak{L}_{ \pm}^{+}:=\mathfrak{R}\left(P_{ \pm}^{+}\right)$are closed subspaces. The set of all operator matrices

$$
\mathscr{W}=\left[\begin{array}{ll}
W_{11} & W_{12} \\
W_{21} & W_{22}
\end{array}\right]
$$

with $W_{11} \in \mathscr{B}\left(\mathfrak{R}_{w}, \mathfrak{L}_{-}^{+}\right), W_{12} \in \mathscr{B}\left(\mathfrak{N}_{\bar{w}}, \mathfrak{L}_{-}^{+}\right), W_{21} \in \mathscr{B}\left(\mathfrak{N}_{w}, \mathfrak{L}_{+}\right)$and $W_{22} \in \mathscr{B}\left(\mathfrak{N}_{\bar{w}}, \mathfrak{L}_{+}\right)$is denoted by $\mathscr{B}_{2}\left(\mathfrak{N}_{w}, \mathfrak{N}_{\bar{w}} ; \mathfrak{L}_{-}^{+}, \mathfrak{L}_{+}\right)$. For $W$ in this set we can define in a natural way the inverse $\mathscr{W}^{-1} \in \mathscr{B}_{2}\left(\mathfrak{L}_{-}^{+}, \mathfrak{L}_{+} ; \mathfrak{N}_{w}, \mathfrak{N}_{\bar{w}}\right)$, if it exists, and the adjoint $\mathscr{W}^{+} \in \mathscr{B}_{2}\left(\mathfrak{L}_{-}, \mathfrak{L}_{+}^{+} ; \mathfrak{N}_{w}, \mathfrak{N}_{\bar{w}}\right)$ :

$$
\mathscr{W}^{+}:=\left[\begin{array}{ll}
W_{11}^{+} & W_{21}^{+} \\
W_{12}^{+} & W_{22}^{+}
\end{array}\right]
$$

Furthermore, we denote by $M_{\mathscr{W}}$ the "Möbius transformation" induced by $\mathscr{W}$ :

$$
M_{\mathscr{W}}(F):=\left(W_{11} F+W_{12}\right)\left(W_{21} F+W_{22}\right)^{-1}
$$

for $F \in \mathscr{B}\left(\mathfrak{N}_{\bar{w}}, \mathfrak{N}_{w}\right)$. For the basic properties of $M_{\mathscr{w}}$, see [2].

If $\tilde{R}$ is a regular generalized resolvent of $T$, then the function $z \mapsto \widetilde{\Omega}(z):=$ $\left.P_{-}^{+} \tilde{R}(z)\right|_{\mathfrak{L}_{+}}$with values in $\mathscr{B}\left(\mathfrak{L}_{+}, \mathfrak{L}_{-}^{+}\right)$is called a $\left(P_{+}, P_{-}\right)$-resolvent of $T$. These resolvents are best studied by means of the so-called $\left(P_{+}, P_{-}\right)$-resolvent matrices. To define the latter, we denote by $\varrho\left(\mathfrak{L}_{+}, \mathfrak{L}_{-}\right)$the set of all $z \in \boldsymbol{C}_{+}$for which

$$
\mathfrak{R}(T-z I) \dot{+} \mathfrak{L}_{+}=\mathfrak{R}(T-\bar{z} I)+\mathfrak{L}_{-}=\mathfrak{H} .
$$


A matrix function $\mathscr{W}$ is called a $\left(P_{+}, P_{-}\right)$-resolvent matrix for $T$ if it has the following properties:

1) $\mathscr{W}$ is defined for a.a. $z \in \varrho\left(\mathfrak{L}_{+}, \mathfrak{L}_{-}\right)$, has values in $\mathscr{B}_{2}\left(\mathfrak{N}_{w}, \mathfrak{N}_{\bar{w}} ; \mathfrak{L}_{-}^{+}, \mathfrak{L}_{+}\right)$ and is meromorphic;

2) $\mathscr{W}(z)^{-1} \in \mathscr{B}_{2}\left(\mathfrak{L}_{-}^{+}, \mathfrak{L}_{+} ; \mathfrak{N}_{w}, \mathfrak{N}_{\bar{w}}\right)$ exists for a.a. $z \in \varrho\left(\mathfrak{L}_{+}, \mathfrak{L}_{-}\right)$;

3) $M_{\mathscr{W}(z)}(F)$ is an operator for all contractive operators $F \in \mathscr{B}\left(\mathfrak{N}_{\bar{w}}, \mathfrak{N}_{w}\right)$ and for a.a. $z \in \varrho\left(\mathfrak{L}_{+}, \mathfrak{L}_{-}\right)$;

4) the formula

$$
\widetilde{\Omega}(z)=M_{\mathscr{W}(z)}(\tilde{F}(z)) \quad \text { for a.a. } z \in \varrho\left(\mathfrak{L}_{+}, \mathfrak{L}_{-}\right)
$$

gives a bijective mapping between the set of all $\left(P_{+}, P_{-}\right)$-resolvents $\widetilde{\Omega}$ of $T$ and the set of all $\tilde{F} \in \mathscr{K}_{+}\left(\mathfrak{N}_{\bar{w}}, \mathfrak{N}_{w}\right)$.

The existence of a $\left(P_{+}, P_{-}\right)$-resolvent matrix is settled by the following result, which generalizes [2], Satz 5.2.

Theorem 2.1. Let $T$ be a closed symmetric linear relation in a Pontrjagin space $\mathfrak{H}$ with $\mathfrak{D}(T) \supset \mathfrak{H}_{-}$and $\mathfrak{n}_{+}(T) \geqq \mathfrak{n}_{-}(T)>0$. Let $\mathfrak{L}_{ \pm}$be closed subspaces of $\mathfrak{H}$ such that $\operatorname{dim} \mathfrak{L}_{ \pm}=\mathfrak{n}_{ \pm}(T)$ and $\varrho\left(\mathfrak{L}_{+}, \mathfrak{L}_{-}\right) \neq \emptyset$. If $P_{ \pm}$are projectors onto $\mathfrak{L}_{ \pm}$, then $T$ has a $\left(P_{+}, P_{-}\right)$-resolvent matrix.

Proof. Choose the $R$ in Theorem 1.1 to be lower canonical. Then with some manipulation one can put (1.1) in the form

$$
\widetilde{\Omega}(z)=\left.P_{ \pm}^{+} \tilde{R}(z)\right|_{\mathfrak{L}_{+}}=M_{\mathscr{W}(z)}(F(z)),
$$

where the components of the desired $\left(P_{+}, P_{-}\right)$-resolvent matrix $\mathscr{W}$ are given by

with

$$
\begin{aligned}
& W_{11}(z):=-P_{-}^{+} R(z) Z(z)^{-1} Y(z)+\left.(w-\bar{w})^{-1} P_{-}^{+} \Gamma_{+}(z)\right|_{\mathfrak{N}_{w}}, \\
& W_{12}(z):=P_{-}^{+} R(z) Z(z)^{-1}-(w-\bar{w})^{-1} P_{-}^{+} \Gamma_{+}(z) F(z), \\
& W_{21}(z):=-Z(z)^{-1} Y(z) \\
& W_{22}(z):=Z(z)^{-1}
\end{aligned}
$$

$$
Z(z):=\left.(I-Y(z) F(z)) \Gamma_{-}(\bar{z})^{+}\right|_{\mathfrak{L}_{+}} .
$$

For details see [2] and [4], where the operator case is considered.

We proceed to characterize all the $\left(P_{+}, P_{-}\right)$-resolvent matrices. For this define

$$
\mathscr{J}:=\left[\begin{array}{cc}
I & O \\
O & -I
\end{array}\right] \in \mathscr{B}_{2}\left(\mathfrak{N}_{w}, \mathfrak{N}_{\bar{w}} ; \mathfrak{N}_{w}, \mathfrak{N}_{\bar{w}}\right)
$$

and denote by $P(z), z \in \varrho\left(\mathfrak{Q}_{+}, \mathfrak{L}_{-}\right)$, the projector onto $\mathfrak{L}_{+}$along $\mathfrak{R}(T-z I)$. This $P$ has a representation

$$
P(z)=\left(\left.\Gamma_{-}(\bar{z})^{+}\right|_{\dot{q}_{+}}\right)^{-1} \Gamma_{-}(\bar{z})^{+},
$$

which implies that $P$ and $Q$,

$$
Q(z):=P_{-}^{+} R(z)(I-P(z)),
$$

are meromorphic in $\varrho\left(\mathfrak{L}_{+}, \mathfrak{L}_{-}\right)$; see [2]. 
The same lines of reasoning as in the operator case show that the $\left(P_{+}, P_{-}\right)$resolvent matrix $\mathscr{W}$ with components (2.1) satisfies the equation

$$
(w-\bar{w}) \mathscr{W}(z) \mathscr{J} \mathscr{W}(\zeta)^{+}=\mathscr{X}(z, \zeta) \mathscr{J} \quad \text { for a.a. } z \in \varrho\left(\mathfrak{L}_{+}, \mathfrak{L}_{-}\right),
$$

where the matrix function $\mathscr{X}$ is given by

$$
\begin{aligned}
& X_{11}(z, \zeta):=\left.P_{-}^{+}\left(Q(z)-Q(\zeta)^{+}\right)\right|_{\mathfrak{I}_{-}}-\left.(z-\bar{\zeta}) Q(z) Q(\zeta)^{+}\right|_{\mathfrak{I}_{-}}, \\
& X_{12}(z, \zeta):=-\left.P_{-}^{ \pm} P(\zeta)^{+}\right|_{\mathfrak{I}_{+}^{+}}-\left.(z-\bar{\zeta}) Q(z) P(\zeta)^{+}\right|_{\mathfrak{I}_{+}^{+}}, \\
& X_{21}(z, \zeta):=-\left.P(z)\right|_{\mathfrak{I}_{-}}+\left.(z-\bar{\zeta}) P(z) Q(\zeta)^{+}\right|_{\mathfrak{I}_{-}}, \\
& X_{22}(z, \zeta):=\left.(z-\bar{\zeta}) P(z) P(\zeta)^{+}\right|_{\mathfrak{L}_{+}^{+}} .
\end{aligned}
$$

Note that (2.2) can be written in the form

$$
\begin{aligned}
& (\bar{w}-w) \mathscr{W}(z) \mathscr{J} \mathscr{W}(\zeta)^{+}=(z-\bar{\zeta})\left[\begin{array}{c}
Q(z) \\
-P(z)
\end{array}\right]\left[\left.Q(\zeta)^{+}\right|_{\mathbb{Q}_{-}} \quad-\left.P(\zeta)^{+}\right|_{\mathfrak{I}_{+}^{+}}\right] \\
& +\left[\begin{array}{cc}
\left.P_{-}^{+}\left(Q(\zeta)^{+}-Q(z)\right)\right|_{\mathfrak{L}_{-}} & -\left.P_{-}^{ \pm} P(\zeta)^{+}\right|_{\mathfrak{I}_{+}^{+}} \\
\left.P(z)\right|_{\mathfrak{L}_{-}} & O
\end{array}\right] .
\end{aligned}
$$

Reasoning further as in [2] (see also [4]) we derive the following characterizations of the $\left(P_{+}, P_{-}\right)$-resolvent matrices.

Theorem 2.2. Let the assumptions of Theorem 2.1 be fulfilled and let $\tilde{\mathscr{W}}: \varrho\left(\mathfrak{I}_{+}, \mathfrak{L}_{-}\right) \rightarrow \mathscr{B}_{2}\left(\mathfrak{N}_{w}, \mathfrak{N}_{\bar{w}} ; \mathfrak{L}_{-}^{+}, \mathfrak{L}_{+}\right)$be meromorphic. Then the following facts are equivalent:

(i) $\tilde{\mathscr{W}}$ is a $\left(P_{+}, P_{-}\right)$-resolvent matrix of $T$;

(ii) there exists a matrix $\mathscr{U} \in \mathscr{B}_{2}\left(\mathfrak{N}_{w}, \mathfrak{N}_{\bar{w}} ; \mathfrak{N}_{w}, \mathfrak{N}_{\bar{w}}\right)$ such that $\mathscr{U} \mathscr{J} \mathscr{U}^{+}=\mathscr{J}$ and $\tilde{\mathscr{W}}(z)=\mathscr{W}(z) \mathscr{U}$ for a.a. $z \in \varrho\left(\mathfrak{Q}_{+}, \mathfrak{Q}_{-}\right)$;

(iii) $\tilde{\mathscr{W}}(z)^{-1} \in \mathscr{B}_{2}\left(\mathfrak{L}_{-}^{+}, \mathfrak{L}_{+} ; \mathfrak{N}_{w}, \mathfrak{N}_{\bar{w}}\right)$ exists and $\tilde{\mathscr{W}}$ satisfies the equation (2.2) for a.a. $z \in \varrho\left(\mathfrak{L}_{+}, \mathfrak{L}_{-}\right)$.

\section{References}

[1] Krein, M. G., and G. K. LANGer: Defective subspaces and generalized resolvents of an Hermitian operator in the space $\Pi_{\varkappa}$ - Functional Anal. Appl. 5, 1971, 217-228.

[2] LANGer, H., and P. SORJONEN: Verallgemeinerte Resolventen hermitescher und isometrischer Operatoren im Pontrjaginraum. - Ann. Acad. Sci. Fenn. Ser. A I 561, 1974, 1-45.

[3] LANger, H., and B. Textorius: On generalized resolvents and $Q$-functions of symmetric linear relations (subspaces) in Hilbert space. - Pacific J. Math. 72, 1977, 135-165.

[4] SoRjonen, P.: Verallgemeinerte Resolventen eines symmetrischen Operators im Pontrjaginraum. - Ber. Univ. Jyväskylä Math. Inst. 15, 1972, 1-58.

[5] Sorjonen, P.: On linear relations in an indefinite inner product space. - Ann. Acad. Sci. Fenn. Ser. A I 4, 1978/1979, 169-192.

University of Jyväskylä

Department of Mathematics

SF-40100 Jyväskylä 10

Finland

Received 3 April 1979 